\title{
$\mathrm{BMJ}$
}

\section{Impact of an informed choice invitation on uptake of screening for diabetes in primary care (DICISION): randomised trial}

\author{
Theresa M Marteau, professor of health psychology, ${ }^{1}$ Eleanor Mann, research assistant in psychology, \\ A Toby Prevost, reader in medical statistics, ${ }^{5}$ Joana C Vasconcelos, medical statistician, ${ }^{2}$ lan Kellar, research \\ psychologist, ${ }^{2}$ Simon Sanderson, senior clinical research associate, ${ }^{2}$ Michael Parker, professor of bioethics \\ and director, ${ }^{3}$ Simon Griffin, assistant director, ${ }^{4}$ Stephen Sutton, professor of behavioural science, ${ }^{2}$ \\ Ann Louise Kinmonth, foundation professor of general practice ${ }^{2}$
}

'King's College London, Psychology Department (at Guy's), Health Psychology Section, Psychology and Genetics Research Group, Guy's Campus, London SE1 9RT 2University of Cambridge Department of Public Health and Primary Care, Cambridge

${ }^{3}$ The Ethox Centre, Division of Public Health and Primary Health Care, University of Oxford

${ }^{4}$ MRC Epidemiology Unit, Institute of Metabolic Science, Box 285, Addenbrooke's Hospital, Cambridge CB2 OQQ

${ }^{5}$ King's College London, Department of Primary Care and Public Health Sciences, Guy's

Campus, London SE1 3QD

Correspondence to: T M Marteau theresa.marteau@kcl.ac.uk

Cite this as: BMJ 2010;340:c2138 doi:10.1136/bmj.c2138

\section{ABSTRACT}

Objective To compare the effect of an invitation promoting informed choice for screening with a standard invitation on attendance and motivation to engage in preventive action.

Design Randomised controlled trial. Setting Four English general practices.

Participants 1272 people aged 40-69 years, at risk for diabetes, identified from practice registers using a validated risk score and invited to attend for screening. Intervention Intervention was a previously validated invitation to inform the decision to attend screening, presenting diabetes as a serious potential problem, and providing details of possible costs and benefits of screening and treatment in text and pie charts. This was compared with a brief, standard invitation simply describing diabetes as a serious potential problem. Main outcome measures The primary end point was attendance for screening. The secondary outcome measures were intention to make changes to lifestyle and satisfaction with decisions made among attenders.

Results The primary end point was analysed for all 1272 participants. 55.8\% (353/633) of those in the informed choice group attended for screening, compared with $57.6 \%(368 / 639)$ in the standard invitation group (mean difference $-1.8 \%, 95 \%$ confidence interval $-7.3 \%$ to $3.6 \% ; \mathrm{P}=0.51$ ). Attendance was lower among the more deprived group (most deprived third $47.5 \% \mathrm{v}$ least deprived third 64.3\%; P 0.001 ). Interaction between deprivation and effect of invitation type on attendance was not significant. Among attenders, intention to change behaviour was strong and unaffected by invitation type.

Conclusions Providing information to support choice did not adversely affect attendance for screening for diabetes. Those from more socially deprived groups were, however, less likely to attend, regardless of the type of invitation received. Further attention to invitation content alone is unlikely to achieve equity in uptake of preventive services.
Trial registration Current Controlled Trials ISRCTN 73125647.

\section{INTRODUCTION}

Population screening programmes are an increasingly important component of health care. Their public health benefits depend on the participation of most people at risk, although each individual has low odds of personal benefit. ${ }^{1}$ Response rates to invitations to attend for medical screening vary by condition and culture and are notably lower among socially deprived groups. ${ }^{2}$ Invitations are traditionally brief and aim to achieve high attendance rates. They most often provide information about the prevalence and severity of the disease to be screened for, and rarely describe the absolute chances of individual benefit or harms. ${ }^{3}$ Policy in the United Kingdom and elsewhere now advocates that participation should reflect "informed choice," which can be defined as individual choices informed by the nature and frequency of individual benefits and harms of screening and actions consistent with participants' values. ${ }^{4}$ This differs from informed consent by its emphasis on choices that are congruent with individuals' values. ${ }^{5}$

This policy change is not yet evident in the content of invitations people routinely receive to participate in screening programmes. This may reflect concern that people reading information about the limited individual benefits and possible harms of screening will feel less positive about screening and not attend. ${ }^{67}$

A particular concern is that providing information to support individual choice might have a differential effect on attendance across social groups, resulting in even lower uptake among more socially deprived people, an example of the "inverse care law." vation is associated with an orientation more towards the present than the future. ${ }^{9}$ Information that the benefits of screening through early diagnosis of disease accrue in the future while the harms and burdens of screening and preventive treatment are more 
immediate may thus reduce attendance, particularly among more socially deprived groups. ${ }^{10}$ This would include more of those at highest risk of disease for whom the benefits of screening might be greatest, ${ }^{11}$ including those at risk of type 2 diabetes, a common, serious condition that can remain undiagnosed until complications occur. ${ }^{12}$ The adverse consequences of screening are likely to be limited ${ }^{13}$ and intensive treatment of screen detected patients is likely to be beneficial. $^{14}$

Screening for type 2 diabetes and cardiovascular risk provides examples of situations where change in behaviour is an important component of preventive action and for which population based screening programmes for people at risk are now being proposed and implemented. ${ }^{1516}$ The evaluation of screening invitations therefore needs to include assessment of effects on motivation to change behaviour if diabetes were diagnosed.

We tested three hypotheses when comparing the informed choice invitation with a standard invitation. Firstly, that attendance at screening for diabetes would be lower after receipt of an informed choice invitation. Secondly, that an interaction exists between the type of invitation and social deprivation, such that attendance would be lower among those from more socially deprived groups. Thirdly, that among those who attend for screening, intentions to change behaviour to reduce the risk of complications, if diabetes were subsequently diagnosed, would be stronger after an informed choice invitation.

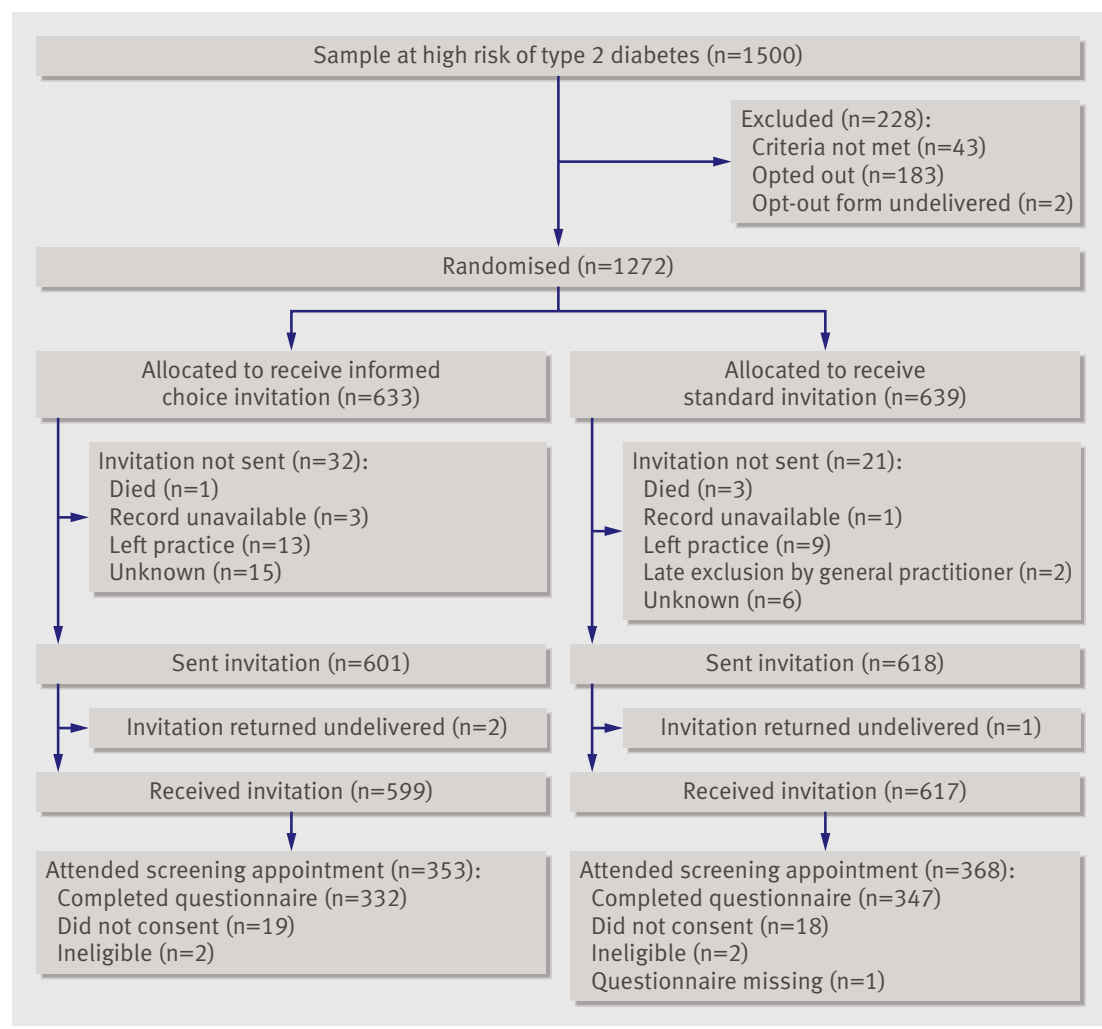

Flow of participants through trial

\section{METHODS}

More details of the trial methods can be found in the published protocol. ${ }^{17} \mathrm{We}$ recruited participants from four practices based in the English counties of Cambridgeshire and Suffolk, each of which varies in degree of deprivation. We assessed the level of social deprivation by using the index of multiple deprivation 2007, which is based on UK postcodes. One practice was from the least deprived postcode fifth, one from the middle fifth, and two from the most deprived fifth. We identified potentially eligible participants from practice registers using a MIQUEST search for routine data, which was used to calculate diabetes risk scores for all adults aged 40-69 without known type 2 diabetes. MIQUEST is the National Health Service licensed software used to extract information from general practice medical records. The validated score used age, sex, body mass index, and prescribed oral antihypertensive and steroid drugs. ${ }^{12}$

No standard approach is used to select groups for screening. Systematic reviews and modelling studies suggest that restricting screening to those at high risk, as we have done, might be the most cost effective approach. ${ }^{18}$ We randomly sampled household clusters in the top $25 \%$ of the risk score distribution to generate the 1500 people approached for the study. The practice in the most deprived area provided data only on body mass index, which we used to identify 407 people at high risk. High body mass index (top fourth) is a good proxy measure of diabetes risk. ${ }^{12}$ We subsequently excluded 43 people with known diabetes who were pregnant, breast feeding, psychotic, or had a life threatening illness. The remainder were sent letters by their general practitioner asking them to return a reply paid opt-out form to their practice within 28 days if they did not wish to receive an invitation for practice based screening. Overall, 183 people opted out $(183 / 1500 ; 12.2 \%)$ and we excluded a further two because consent letters were returned undelivered. Those who opted out were older than those who participated, but similar in terms of deprivation and sex.

\section{Randomisation}

We randomly assigned 1272 participants to receive one of two invitations to screening: an extended invitation designed to facilitate informed choice, or a short, standard invitation. Members from the same household were assigned to receive the same invitation type (mean cluster size 1.14). Randomisation was done at once for all participants in each practice and was undertaken by the study statistician from a central site. For each practice separately, allocations were generated simultaneously in a batch by random numbers using Excel spreadsheet software, stratifying by number of participants in the household.

We cluster randomised 281 people with at least one other participant from the same household. Clinical and trial staff taking measurements and entering data were unaware of the study arm to which participants had been assigned. Fifty six participants did not receive an invitation: 53 were not sent an invitation and three 
Table 1|Personal characteristics of participants randomised to receive an invitation for screening

\begin{tabular}{lccc} 
Variable & Overall $(\mathrm{n}=1272)$ & $\begin{array}{c}\text { Informed choice } \\
\text { invitation arm }(\mathrm{n}=633)\end{array}$ & $\begin{array}{c}\text { Standard invitation } \\
\text { arm }(\mathrm{n}=639)\end{array}$ \\
\% (No) women & $45.5(579)$ & $47.6(301)$ & $43.5(278)$ \\
\hline Mean (SD) age (years) & $55.5(8.1)$ & $55.4(8.0)$ & $55.6(8.2)$ \\
\hline Mean (SD) body mass index* & $33.6(6.0)$ & $33.8(6.0)$ & $33.4(6.0)$ \\
\hline$\%($ No) obese* & $69.1(817)$ & $69.4(413)$ & $68.8(404)$ \\
\hline$\%(N o)$ prescribed antihypertensives† & $44.3(383)$ & $43.6(187)$ & $45.0(196)$ \\
\hline $\begin{array}{l}\text { Mean (SD) index of multiple deprivation } \\
2007 \text { scoreł }\end{array}$ & $20.1(10.3)$ & $20.1(10.0)$ & $20.0(10.5)$
\end{tabular}

*Based on 595 participants in the informed choice arm and 587 in the standard invitation arm.

†Based on 429 participants in the informed choice arm and 436 in the standard arm from three practices, unavailable from one practice.

†Score combines several indicators, chosen to cover a range of economic, social, and housing issues at district level, into a transformed index from 0-100. UK average is 21.67; eastern England regional average is 15.40. Top $10 \%$ deprived areas have average scores above 50 .

invitations were returned undelivered. Among those attending for screening, consent to participate further was obtained at screening appointments.

\section{Standard invitation}

The standard invitation reflected those commonly used to invite people to screening for diabetes and coronary heart disease $\mathrm{e}^{219}$ and was based on examples used in a trial and a national pilot of screening. ${ }^{2021}$

The text stated that screening was offered because the participant may have a higher chance of developing type 2 diabetes and that diabetes is a common, often undiagnosed condition with serious long term consequences (see web extra).

\section{Informed choice invitation}

The informed choice invitation contained the same information as the standard invitation, along with information about the risk of diabetes and its complications and the consequences of screening and treatment, including a description of the possible harms associated with attending for screening. The invitations drew on best evidence and were based on the UK General Medical Council guidelines for consent. ${ }^{22}$ These recommend that the following information is presented when obtaining consent for screening: the purpose of screening; details of diagnosis and prognosis with and without treatment; and the probability of benefits and risks, presented with an emphasis on patient choice (see web extra).

Drawing on these guidelines, the draft invitation text was developed iteratively using "think-aloud" methods with volunteers. ${ }^{23}$ The method requires respondents to verbalise their thoughts while reading the invitation and has typically been used to examine decision making. ${ }^{2425}$ In this case we used the verbal reports to ascertain where information was not being understood as intended and then refined the text by using readability tools. ${ }^{26}$ By using a decisional balance sheet we encouraged participants to make a choice that reflected their values. Texts of both invitations were comprehensible to those with reading ages of 11 and above. ${ }^{27}$
These invitations were developed and evaluated in a study in which 417 adults without known diabetes were randomised to receive one of the invitations. Levels of informed choice were significantly higher two weeks after receipt of the informed choice invitation compared with the standard invitation $(42.9 \%$ v 11.2\%; difference $31.7 \%$, 95\% confidence interval $22.5 \%$ to $40.5 \%)^{27}$ - an outcome largely attributable to higher levels of knowledge.

\section{Procedure}

Participants were invited by letter sent from their general practice to attend pre-assigned screening appointments for diabetes with a research nurse at their practice. Appointments took about 20 minutes and were done according to a written clinical protocol. The research nurse recorded attendance as participants arrived and sought their written consent to be screened for diabetes. Consent was also sought to complete research questionnaires at the consultation and by post four weeks later. Thirty seven participants $(5 \%)$ declined consent to complete questionnaires and four met the exclusion criteria (three had diagnosed diabetes and one was judged unable to give consent). The nurse carried out a finger prick random capillary blood glucose test using a glucose analyser based on the glucose dehydrogenase reaction (HemoCue B-glucose analyser; HemoCue, Angelholm, Sweden). The optimal screening test remains uncertain, ${ }^{18}$ but suitable tests certainly include capillary blood samples in the non-fasted state, as used in this trial.

The stability of the analyses was checked daily and an external calibration with the quality assurance scheme was undertaken monthly. Participants were given their results immediately, along with brief standardised advice about healthy lifestyle. Those with blood glucose levels of $5.5 \mathrm{mmol} / 1$ or more were invited to return for a fasting capillary blood glucose test and a full cardiovascular risk assessment, carried out by the research nurse. The nurse measured the fasting blood glucose level, full lipid profile, height, weight, blood pressure, and cardiovascular risk score. Patients were given further standardised advice on lifestyle, and those with fasting blood glucose levels of $6.1 \mathrm{mmol} / \mathrm{l}$ or more were referred to their general practitioner for further testing and confirmation of a diagnosis of diabetes. Four weeks after the appointment for the random glucose blood test, attenders were posted a follow-up questionnaire, which included a measure of satisfaction with their decision.

\section{Outcome measures}

The primary outcome measure was attendance for screening. This was recorded by the research nurse and measured as the proportion of those invited who attended for screening. The secondary outcome measures were intention to make changes to lifestyle and satisfaction with decisions made.

We assessed the intention to make changes to lifestyle if diabetes were subsequently diagnosed among attenders using the arithmetic mean of three intention 
items relating to treatment adherence, reducing fat intake, and increasing physical activity (Cronbach's $\alpha=0.58)$. Items were rated on a 7 point scale - for example, "If the tests show that you definitely do have diabetes, how likely is it that you will increase the amount of physical activity that you do over the next 3 months? "( 1 extremely unlikely to 7 extremely likely).

Satisfaction with the decision was assessed among attenders at four weeks by postal questionnaire using the mean of three items (Cronbach's $\alpha=0.92$ ) - for example, "How sure are you that the decision you made was the right one for you?" ( 1 not at all to 7 extremely).

\section{Statistical analysis}

We determined that a sample size of 1200 participants would provide $90 \%$ power to detect a $10 \%$ difference between arms in the proportion attending for screening using a $\chi^{2}$ test at the $5 \%$ level of significance. Assuming an average attendance of $65 \%{ }^{2}$ gave an expectation of 780 attenders. Full response to the initial questionnaire allowed $80 \%$ power to detect a small effect size (0.2 standardised difference) between arms in the intention measure using a $t$ test at the $5 \%$ level of significance.

We tested the first hypothesis by comparing attendance rates in the informed choice and standard invitation arms using the $\chi^{2}$ test in an intention to treat analysis. For the second hypothesis, we used logistic regression to test for moderating effects of social deprivation on the impact of invitation type on attendance by including an interaction between arm and social deprivation index. We carried out per protocol analyses for both these hypotheses, which excluded participants who did not receive an invitation $(n=56)$ and those randomised but subsequently found to meet exclusion criteria $(n=4)$. For the third hypothesis we used a $t$ test to assess the difference between attenders' intentions to change behaviour in the informed choice arm compared with standard invitation arm. Using a test we also compared the groups for satisfaction with the decision. All tests were two tailed and assessed at the $5 \%$ level of significance. Further analyses that allowed for clustering of participants in randomised households were defined as secondary owing to the anticipated small mean cluster size. The results of these did not materially affect the primary results or conclusions.

\section{RESULTS}

Recruitment to the trial started in November 2006 and ended in July 2008. We analysed screening attendance for all 1272 randomised participants (figure). Baseline characteristics of the two groups were similar (table 1). Those invited for screening were middle aged men and women, many of whom were obese or taking antihypertensive drugs. Overall, 721 (56.7\%) of the randomised participants attended. Compared with attenders, non-attenders were younger, had a higher body mass index, lived in more deprived areas, and were less likely to be prescribed antihypertensive drugs (table 2).

Attendance at screening for diabetes was not significantly lower after an informed choice invitation compared with a standard invitation: $56.7 \%$ v $57.6 \%$ (difference $-1.8 \%, 95 \%$ confidence interval $-7.3 \%$ to $3.6 \%, \mathrm{P}=0.51$; table 3 ). The interaction between type of invitation and social deprivation was not significant. However, attendance fell with increasing deprivation $(64.3 \%$ in the lowest third $v 47.5 \%$ in the highest third, $\mathrm{P}<0.001)$. Per protocol analyses did not affect these findings.

Overall, 680/721 participants (94.3\%) agreed to answer questionnaires at first attendance and 525/721 $(72.8 \%)$ returned their four week follow-up questionnaires. Intention to make changes to lifestyle at first attendance was unaffected by screening invitation: mean intention to change behaviour if diabetes were to be diagnosed was 5.84 (SD 1.09) in the informed choice arm and 5.84 (SD 1.04) in the standard invitation arm. Satisfaction with the decision to attend (measured in the four week follow-up questionnaire) was high and unaffected by screening invitation: mean 6.47 (SD 0.84) in the informed choice arm and 6.48 (SD 0.87) in the standard invitation arm (difference $-0.01,95 \%$ confidence interval -0.17 to $0.16 ; \mathrm{P}=0.90$ ).

No adverse events were recorded during the trial.

\section{DISCUSSION}

An informed choice invitation for diabetes screening did not significantly reduce rates of attendance compared with a standard letter. No interaction was present between type of invitation and levels of deprivation.

Table 2 |Characteristics of non-attenders and attenders pooled over trial arms

\begin{tabular}{|c|c|c|c|}
\hline \multirow[b]{2}{*}{ Variable } & \multicolumn{2}{|c|}{ Overall } & \multirow[b]{2}{*}{ Pvalue* } \\
\hline & Non-attenders $(n=551)$ & Attenders $(n=721)$ & \\
\hline$\%$ (No) women & $42.8(236)$ & $47.6(343)$ & 0.092 \\
\hline Mean (SD) age (years) & $53.0(7.9)$ & $57.4(7.8)$ & $<0.001$ \\
\hline Mean (SD) body mass index $\dagger$ & $34.8(6.3)$ & $32.7(5.6)$ & $<0.001$ \\
\hline \% (No) prescribed drugs $\ddagger$ & $46.2(150)$ & $53.0(286)$ & 0.052 \\
\hline$\%$ (No) prescribed antihypertensives & 39.7 (129) & $47.0(254)$ & 0.035 \\
\hline Mean (SD) index of multiple deprivation 2007 score & $21.6(10.6)$ & $18.8(9.9)$ & $<0.001$ \\
\hline
\end{tabular}

*Unpaired $t$ test for age, body mass index, and index of multiple deprivation 2007 score; Pearson $x^{2}$ test for other variables.

†Based on 513 non-attenders and 669 attenders.

‡Based on 325 non-attenders and 540 attenders. 
Table $3 \mid$ Attendance at diabetes screening after receipt of informed choice invitation or standard invitation, grouped by social deprivation thirds. Values are percentages (numbers) unless stated otherwise

\begin{tabular}{lcccc} 
Deprivation* & Overall $(\mathrm{n}=1272)$ & $\begin{array}{c}\text { Informed choice invitation } \\
(\mathrm{n}=633)\end{array}$ & $\begin{array}{c}\text { Standard invitation } \\
(\mathrm{n}=639)\end{array}$ & $\begin{array}{c}\text { \% difference in uptake } \\
\text { between arms }\end{array}$ \\
Overall & $56.7(721)$ & $55.8(353)$ & $57.6(368)$ & $-1.8(-7.3$ to 3.6); $\mathrm{P}=0.51$ \\
\hline Lowest third ((13.7) & $64.3(272)$ & $64.3(133)$ & $64.4(139)$ & $-0.1(-9.2$ to 9.0$)$ \\
\hline Middle third (13.7-23.5) & $58.5(244)$ & $55.8(116)$ & $61.2(128)$ & $-5.5(-14.9$ to 4.0$)$ \\
\hline Highest third (223.5) & $47.5(205)$ & $47.7(104)$ & $47.2(101)$ & $0.5(-8.9$ to 9.9)
\end{tabular}

$\mathrm{P}=0.50$ for interaction between arm and index of multiple deprivation 2007 on uptake.

*Index of multiple deprivation 2007 third.

Social deprivation did, however, strongly predict attendance. Regardless of invitation type, those from more deprived areas were less likely to attend for screening. Among those attending for screening, the type of invitation did not affect their intentions to change their behaviour to reduce risks were diabetes subsequently diagnosed. Among both groups intentions were strong. Similarly, satisfaction with the decision to attend for screening was high in both groups.

\section{Meaning of the study results}

We considered several possible explanations for our main finding. Firstly, although the informed choice invitation was longer and contained more information than the standard one, recipients may have treated the two invitations as similar. If, for example, they had gone no further than the opening paragraph, they would have read the same information as that presented in the standard invitation. Secondly, the provision of a prearranged screening appointment may have been regarded as an implicit recommendation for screening, over-riding any influence of different invitations. Thirdly, recipients may have gained more information from the informed choice invitation, but this knowledge did not affect attendance. This may be because they perceived the harms associated with screening to be negligible or the benefits to be very good. In support of this, in our pilot study we found that the informed choice invitation increased knowledge about screening for diabetes but did not affect attitudes, which were consistently positive, or intentions to attend for screening. ${ }^{27}$ Other studies have found knowledge about screening to be a weak predictor of attendance and to have little or no association with attitudes towards attending for screening. ${ }^{2728} \mathrm{In}$ contrast, attitudes towards screening have been found to be moderately strong predictors of attendance. ${ }^{6}$

The gradient in uptake with social advantage has been observed previously. ${ }^{2930}$ This gradient may reflect differences in people's attitudes or differences in their enactment of similar positive intentions according to their different social circumstance. In our pilot study, individual attitudes and intentions to attend for diabetes screening were positive regardless of social deprivation, ${ }^{27}$ a finding supported by other studies. ${ }^{31}$ This suggests a social patterning not to intentions but to the enactment of intentions, perhaps reflecting practical barriers to keeping appointments.
Implications for clinicians and policy makers

Taken together the evidence from this trial and other studies suggests that providing information to support choice does not adversely affect attendance for screening: written information can increase knowledge about screening but this does not necessarily alter uptake or increase the likelihood that choices are more value consistent - that is, informed. ${ }^{27283233}$ But although we found no evidence of harm, there was little to suggest any quantitative benefit. The informed choice invitations may have resulted in people being better informed which, from the perspective of individual autonomy, is a benefit. People with better knowledge are less anxious when recalled for further tests, ${ }^{34}$ so such invitations may be preferred, although anxiety is not a major concern in screening for diabetes. ${ }^{13}$

Importantly, our findings raise further questions about the equity of programmes that fail to engage those who are more socially deprived and therefore with lower health and life chances. Overall attendance was low, albeit similar to a national pilot screening programme in deprived areas in England. ${ }^{35}$ Compared with attenders, non-attenders were not only more likely to be from more deprived areas but also to have a higher body mass index, despite being younger, and less likely to be prescribed antihypertensive drugs. A mix of opportunistic approaches to screening (for example, during planned reviews of drugs for hypertension) combined with a range of systematic approaches that go beyond repeated invitation letters, however elaborate, may be most effective in creating environments that enable people to enact their choices. These include visiting patients at home ${ }^{36}$ and training receptionists to telephone and talk to patients. ${ }^{37}$ The effectiveness and appropriateness of these and other interventions merit further consideration given the large gap in screening uptake between the most and least deprived groups, which is not accompanied by evidence of a difference in motivation or preference, both of which are uniformly high.

\section{Strengths and limitations of the study}

The trial design was rigorous for several reasons. We defined a real world at risk population through primary care, and $87.3 \%$ of the eligible population were randomised and participated. Both invitations were delivered in the same way and were similar for readability. Attendance was measured reliably for every participant, and those recording it were blind to the 


\begin{tabular}{|l|}
\hline WHAT IS ALREADY KNOWN ON THIS TOPIC \\
\hline Health policies now hold that participation in screening \\
programmes should reflect "informed choice" \\
Informed choice requires the communication of the chances \\
of individual benefit and harm of screening, and actions \\
consistent with participants' values \\
Such choice may deter attendance, particularly among \\
socially disadvantaged groups, and so increase inequalities \\
in health \\
WHAT THIS STUDY ADDS \\
Providing information to support choice does not adversely \\
affect attendance for screening \\
More socially deprived groups are, however, less likely to \\
attend regardless of type of invitation \\
Those designing and implementing screening and other \\
prevention programmes need to consider how to most \\
effectively overcome inequities in use of screening
\end{tabular}

study arm. Randomisation was at the level of the individual, hence potential confounders were equally distributed across trial groups despite the small number of participating practices. The results confirm the findings of other trials, that informed choice invitations have no impact on attendance. However, these trials either failed to assess behaviour objectively ${ }^{32}$ or recruited only those who had opted into the study ${ }^{33}$ as opposed to using an opt-out method as here, which is preferred for minimising bias. ${ }^{38}$

The trial has several limitations. Our measure of social deprivation was based on postcode, which was available for all those invited. This had limited precision for individual measurement. We did not recruit many participants in the most deprived $10 \%$ of the distribution. Despite these limitations, the measures were sensitive enough for a strong gradient in attendance to be shown across levels of deprivation. From an ethical point of view it was imperative to recruit eligible participants using an opt-out form for those not wishing to receive an invitation for practice based screening. This is unlikely to have influenced the generalisability of the findings or the trial group comparisons, given the small number who opted out and the limited information provided compared with the informed choice and standard invitations to attend screening. The findings are, however, limited in the extent to which they may be generalised to other invitations for diabetes screening as well as invitations for other types of screening.

Although the invitation materials were pilot tested and found to increase knowledge $\mathrm{e}^{27}$ we did not extend the information provided using web based supplements. Providing these would have allowed patients to gain further information in a preferred format, including the generation of a personalised risk score. ${ }^{39}$ Additionally, web based systems allow monitoring of the information accessed, something not possible with paper based materials. Tailored approaches can be more effective in generating change in behaviour, ${ }^{40}$ but whether they more effectively facilitate informed choices is unknown. Furthermore, since web access is socially patterned, its use was not appropriate for the current trial. In 2009, 21\% of UK adults had never used the internet, ${ }^{41}$ a group disproportionately represented among the participants of particular interest in this trial-namely, those who are socially deprived.

Unanswered questions and future research

The generalisability of these findings to other screening programmes is unknown. The harms that can arise from screening for diabetes were described in the invitation as comprising worry and false reassurance, which participants may not consider serious. By contrast, participation in other screening programmes can entail serious physical harms from subsequent invasive testing such as colonoscopy, or treatment such as prostatectomy. The impact of increasing knowledge about the limited individual benefit and potential harms of treatment after screening has been studied most extensively in the context of screening for prostate cancer. The evidence is mixed: in one review increasing knowledge had no impact ${ }^{28}$ but in another it reduced uptake. ${ }^{42}$ Studies are needed to compare the impact of presenting uncertainty about individual benefit and harm across screening programmes with different types and levels of harm and benefit. Our findings also contribute to a broader debate about the most effective and acceptable ways to communicate about screening programmes. ${ }^{43}$ Furthermore, the study hypotheses focused on attendance, and little information was collected on non-attenders. Future studies need to focus on non-attenders to understand better their reasons for non-attendance as a basis for enabling informed choices in this group.

\section{Conclusions}

We observed no conflict between efforts to achieve informed choices and attendance for a population health screening programme for diabetes. Attendance was low, however, and socially deprived groups, who are most vulnerable to disease, were least likely to attend. Further attention to only the content of an invitation is unlikely to achieve equity in uptake of preventive services. Those designing and implementing screening as well as other prevention programmes need to consider how to overcome these inequities most effectively.

We thank study participants and the staff at Bretton Medical Practice, Peterborough; Old Fletton Surgery, Peterborough; The Rookery Medical Centre, Newmarket; and Thorney Medical Practice, Peterborough; the nurses who conducted the screening clinics led by Marian Bosman; the Medical Research Council field epidemiology team; Nicola Popplewell, Helen Morris, Kate Williams, and Rachel Crockett for their contribution to the development of the study and the materials; and Irwin Nazareth, Graham Watt, and Dan Mason for their contribution to discussion of the results. ALK, TMM, SG, and SS are founder members of the National Institute for Health Research School for Primary Care Research. ALK and SS are National Institute of Health Research senior investigators. SG receives support from the Department of Health National Institute for Health Research programme grant funding scheme (RP-PG-0606-1259). The General Practice and Primary Care Research Unit is supported by 
funds from the National Institute for Health Research. IK is supported by a Medical Research Council co-op grant (RG35746).

Contributors: TMM and ALK defined the research question. TMM, ALK, SS ATP, IK, and SG participated in the design of the trial and intervention. SS, $\mathrm{EM}$, and SG directed the intervention team; and EM directed the trial coordination team. ATP wrote the statistical analysis plan and JV did the statistical analyses. All authors participated in the acquisition and analysis of data and critical revision of the manuscript, have seen and approved the final version, had full access to all study data, and were jointly responsible for the decision to submit for publication. TMM is guarantor for the paper.

Funding: This trial was funded by the Wellcome Trust (grant No 076838 "Didactic versus informed choice invitations: balancing public health benefits and individual choice" principal investigator TMM). The funding body had no role in study design, data collection, analysis, interpretation, or writing of the report.

Competing interests: All authors have completed the unified competing interest form at www.icmje.org/coi_disclosure.pdf (available on request from the corresponding author). SG has received honorariums from Eli Lilly, GlaxoSmithKline, Merck, Sharp \& Dohme, Colgate Palmolive, Unilever, the University of Western Ontario, and the National Health Service for undertaking lectures at educational meetings not directly related to the topic of this paper. His second class rail travel costs for attending Department of Health meetings concerning the NHS health check were reimbursed by the Department of Health.

Ethical approval: This study was approved by the Cambridgeshire 1 research ethics committee (reference 06/Q0104/17).

Data sharing: Study materials and documents described in the General Medical Council guidelines for consent are available from the corresponding author at theresa.marteau@kcl.ac.uk.

1 Rose G. Sick individuals and sick populations. Int J Epidemiol 2001;30:427-32.

2 Wood DA, Kinmonth AL, Davies GA, Yarwood J, Thompson SD, Pyke SDM, et al. Randomised controlled trial evaluating cardiovascular screening and intervention in general practice: principal results of British family heart study. BMJ 1994;308:313-20.

3 Jorgensen K, Gotzsche PC. Content of invitations for publicly funded screening mammography. BMJ 2006;332:538-41.

4 Marteau TM, Dormandy E, Michie S. A measure of informed choice. Health Expect 2001;4:99-108.

5 Marteau TM. Informed choice: a construct in search of a name. In: Edwards A, Elwyn G, eds. Shared decision-making in health care. Oxford University Press, 2009:87-94.

6 Cooke R, French D. How well do the theory of reasoned action and theory of planned behaviour predict intentions and attendance at screening programmes? A meta-analysis. Psychol Health 2008;23:745-65.

7 Marteau TM, Hankins M, Collins B. Perceptions of risk of cervical cancer and attitudes towards cervical screening: a comparison of smokers and non-smokers. Fam Pract 2002;19:18-22.

8 Tudor Hart J. The inverse care law. Lancet 1971;1:405-12.

9 Crockett R, Wienmann J, Hankins M, Marteau TM. Time orientation and health-related behaviour: measurement in general population samples. Psychol Health 2009;24:18.

10 Orbell S, Hagger M. Temporal framing and the decision to take part in type 2 diabetes screening: effects of individual differences in consideration of future consequences on persuasion. Health Psychol 2006;25:537-48.

11 Raffle AE. Information about screening - is it to achieve high uptake or to ensure informed choice? Health Expect 2001;4:92-8.

12 Griffin SJ, Little PS, Hales CN, Kinmonth AL, Wareham NJ. Diabetes risk score: towards earlier detection of type 2 diabetes in general practice. Diabetes Metab Res Rev 2000;16:164-71.

13 Adriaanse CA, Snoek F). The psychological impact of screening for type 2 diabetes. Diabetes Metab Res Rev 2006;22:20-5.

14 Holman RR, Paul SK, Bethel MA, Matthews DR, Neil HA. 10-year follow-up of intensive glucose control in type 2 diabetes. $N$ Engl J Med 2008;359:1577-89.

15 Department of Health. Putting prevention first. Vascular checks: risk assessment and management. Stationery Office, 2008.

16 Kohro T, Furui Y, Mitsutake N, Fujii R, Morita H, Oku S, et al. The Japanese national health screening and intervention program aimed at preventing worsening of the metabolic syndrome. Int Heart J 2008;49:193-203.

17 Mann E, Prevost AT, Griffin S, Kellar I, Sutton S, Parker M, et al. Impact of an informed choice invitation on uptake of screening for diabetes in primary care (DICISION): trial protocol. BMC Public Health 2009;9:63.

18 Waugh N, Scotland G, McNamee P, Gillett M, Brennan A, Goyder E, et al. Screening for type 2 diabetes: literature review and economic modelling. Health Technol Assess 2007;11:iii-iv,ix-xi,1-125.

19 Muir J, Mant D, Jones L, Yudkin P, on behalf of the Imperial Cancer Research Fund OXCHECK study group. Effectiveness of health checks conducted by nurses in primary care: results of the OXCHECK study after one year. BMJ 1994;308:308-12.

20 Goyder EC. Screening for and prevention of type 2 diabetes. BMJ 2008;336:1140-1.

21 Echouffo-Tcheugui JB, Simmons RK, Williams KM, Barling RS, Prevost AT, Kinmonth AL, et al. The ADDITION-Cambridge trial protocol: a cluster-randomised controlled trial of screening for type 2 diabetes and intensive treatment for screen-detected patients. BMC Public Health 2009;9:136.

22 General Medical Council. Seeking patients' consent: the ethical considerations. GMC, 1998

23 Gilhooley C, Green C. Protocol analysis: theoretical background. In: Richardson JTE, ed. Handbook of qualitative research methods for psychology and the social sciences. BPS Books, 1996:43-54.

24 Patel VL, Arocha JF, Diermeier M, How J, Mottur-Pilson C. Cognitive psychological studies of representation and use of clinical practice guidelines. Int J Med Inf 2001;63:147-67.

25 Skaner Y, Backlund L, Montgomery H, Bring J, Strender LE. General practitioners' reasoning when considering the diagnosis heart failure: a think-aloud study. BMC Fam Pract 2005;6:4.

26 Flesch [Java program]. 2.0 version, 2007.

27 Kellar I, Sutton S, Griffin S, Prevost AT, Kinmonth AL, Marteau TM. Evaluation of an informed choice invitation for type 2 diabetes screening. Patient Educ Couns 2008;72:232-8

28 Fox R. Informed choice in screening programmes: do leaflets help? A critical literature review. J Public Health 2006;28:309-17.

29 Kim LG, Thompson SG, Marteau TM, Scott RA. Screening for abdominal aortic aneurysms: the effects of age and social deprivation on screening uptake, prevalence and attendance at follow-up in the MASS trial. J Med Screen 2004;11:50-3.

30 McCaffery K, Wardle J, Nadel M, Atkin W. Socioeconomic variation in participation in colorectal cancer screening. I Med Screen 2002;9:104-8.

31 Dormandy E, Michie S, Hooper R, Marteau T. Informed choice in antenatal Down syndrome screening: a cluster-randomised trial of combined versus separate visit testing. Patient Educ Couns 2006;61:56-64.

32 Trevena LJ, Irwig L, Barratt A. Randomized trial of a self-administered decision aid for colorectal cancer screening. J Med Screen 2008;15:76-82.

33 Mathieu E, Barratt A, Davey HM, McGeechan K, Howard K, Houssami N. Informed choice in mammography screening: a randomized trial of a decision aid for 70-year-old women. Arch Intern Med 2007;167:2039-46.

34 Shaw C, Abrams K, Marteau T. Psychological impact of predicting individuals' risks of illness: a systematic review. Soc Sci Med 1999:49:1571-98.

35 Goyder E, Wild S, Fischbacher C, Carlisle J, Peters J. Evaluating the impact of a national pilot screening programme for type 2 diabetes in deprived areas of England. Fam Pract 2008;25:370-5.

36 Marsh GN, Channing DM. Narrowing the health gap between a deprived and an endowed community. BMJ 1988;296:173-6.

37 Atri J, Falshaw M, Gregg R, Robson J, Omar RZ, Dixon S. Improving uptake of breast screening in multiethnic populations: a randomised controlled trial using practice reception staff to contact nonattenders. BMJ 1997:315:1356-9.

38 Gallo C, Perrone F, De Placido S, Giusti C. Informed versus randomised consent to clinical trials. Lancet 1995;346:1060-4

39 Kreuter MW, Bull FC, Clark EM, Oswald DL. Understanding how people process health information: a comparison of tailored and nontailored weight-loss materials. Health Psychol 1999:18:487-94.

40 Noar SM, Benac CN, Harris MS. Does tailoring matter? Meta-analytic review of tailored print health behavior change interventions. Psychol Bull 2007;133:673-93.

41 Office for National Statistics. Internet access: Households and individuals. Stat Bull 28 Aug;2009.

42 O'Connor AM, Stacey D, Entwistle V, Llewellyn-Thomas H, Rovner D, Holmes-Rovner M, et al. Decision aids for people facing health treatment or screening decisions. Cochrane Database Syst Rev 2003; (2):CD001431

43 Entwistle VA, Carter SM, Trevena L, Flitcroft K, Irwig L, McCaffery K, et al. Communicating about screening. BMJ 2008;337:a1591.

Accepted: 27 January 2010 